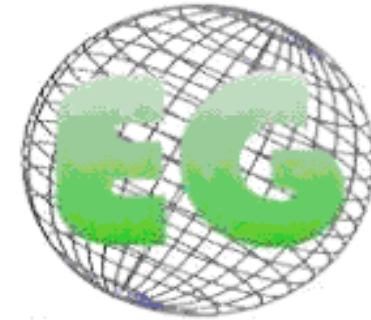

ISSN 1695-6141

$\mathrm{N}^{\circ} 21$

\title{
USO DE PLANTAS MEDICINALES POR PACIENTES ONCOLÓGICOS Y FAMILIARES EN UN CENTRO DE RADIOTERAPIA
}

UTILIZACÃO DE PLANTAS MEDICINAIS POR PACIENTES ONCOLÓGICOS E FAMILIARES NUM CENTRO DE RADIOTERAPIA

*Vanini, M., " ${ }^{* *}$ Barbieri, RL., "**Heck, RM., "**Schwartz, E.

*Enfermeira, mestranda do Programa de Pós Graduação da Faculdade de Enfermagem e Obstetrícia Universidade Federal de Pelotas (UFPEL). ${ }^{* *}$ Doutora em Genética e Biologia Molecular. Pesquisadora da Embrapa Clima Temperado. ${ }^{* * *}$ Professora Adjunta da Faculdade de Enfermagem e Obstetrícia da Universidade Federal de Pelotas.Brasil.

Palavras chave: Neoplasia, Fitoterapia, Plantas medicinais, Enfermagem.

Palabras clave: Neoplasia, Fitoterapia, Plantas medicinales, Enfermería.

Keywords: Neoplasm, Phytotherapy, Medicinal plants, Nursing.

\section{RESUMEN}

El cáncer es un problema de salud pública y una enfermedad que sigue sin la certeza de curación, adquirendo así el estigma de terminal. Además de los tratamientos convencionales se percibe la presencia de algunas terapias alternativas entre pacientes con dolencias crónicas.. El objetivo de este trabajo es investigar el uso de plantas como terapia complementaria para pacientes con cáncer que frecuentan un centro de radioterapia en una clínica pública en el sur de Río Grande do Sul. La metodología es cualitativa, se abordó a los pacientes y familiares, a través de entrevistas semiestructuradas, en el periodo de enero a diciembre de 2006. Los resultados indican el uso generalizado de terapias complementarias para pacientes con cáncer que reciben radioterapia, entre los citados tratamientos la utilización de plantas medicinales como Tabebuia avelanedae, Aloe sp, Matricaria chamomilla, Achyrocline satureioides. Frente a la información, se recomienda a los profesionales de la salud que trabajan directamente con las personas con cáncer atención para proporcionar información segura y cualificada acerca de estas terapias complementarias.

\section{RESUMO}

O câncer é considerado um problema de saúde pública e uma patologia que ainda continua sem a certeza de cura, adquirindo assim o estigma de terminalidade. Percebe-se que além dos tratamentos convencionais estão presentes também algumas terapias alternativas entre pacientes com doenças crônicas. O objetivo desse trabalho é relatar o uso de plantas, como terapia complementar, por 
pacientes oncológicos que realizam radioterapia em um ambulatório pertencente a uma Universidade Pública ao Sul do Rio Grande do Sul. A metodologia é qualitativa e abordou pacientes e familiares que freqüentavam um centro de radioterapia através de entrevistas semi-estruturadas, no período entre janeiro a dezembro de 2006. Os resultados mostram a ampla utilização de terapias complementares pelos pacientes portadores de câncer que realizam radioterapia, dentre as terapias citadas surgiu a utilização de plantas medicinais como cavalinha, ipê roxo, casca de nozes moída, babosa, marcela e camomila. Constatamos que os profissionais de saúde que trabalham diretamente com portadores de câncer devem ser qualificados para oferecer informações seguras a respeito dessas terapias complementares.

\section{ABSTRACT}

Cancer is considered a public health problem and a pathology which is still without the certainty of cure, thus acquiring the stigma of terminality. It is realized that in addition to conventional treatments there also exist some alternative therapies among patients with chronic diseases. The aim of this study was to report the use of plants as complementary therapy for oncology patients undergoing radiotherapy at a clinic belonging to a State University of Southern Brazil, Rio Grande do Sul State. This is a qualitative research based on semi-structured interviews carried out with patients and family members who frequented a radiotherapy center between January and December 2006. The findings show the wide use of complementary therapies for cancer patients undergoing radiotherapy. The therapies mentioned included use of the following medicinal plants: horsetail, pink trumpet tree, crushed nutshell, Aloe vera, chamomile and marcela. We recommend that the health professionals who work directly with people with cancer should be qualified to offer secure information about these complementary therapies.

\section{INTRODUCCIÓN}

La Organización Mundial de la Salud (OMS) estima que 7,6 millones de personas murieron de cáncer en 2005 y que 84 millones morirán en diez años si algo no se hace. Más del 70\% de las muertes ocurren en países en desarrollo y del Tercer Mundo, donde los recursos para la prevención, diagnóstico y tratamiento de la enfermedad son limitados o inexistentes. Esta situación caracteriza el cáncer como un problema de salud pública y justifica los movimientos recientes en torno a una estrategia global de control. La estimativa de incidencia de cáncer en Brasil muestra que aproximadamente 470 mil nuevos casos de la enfermedad deberán ocurrir en el país entre este y el próximo año (1).

A pesar del innegable desarrollo tecnológico y científico, este tipo de patologías aún continúa sin la certeza de cura, adquiriendo el estigma de terminal (2). A través de la literatura se sabe que la quimioterapia, radioterapia, hormonoterapia, inmunoterapia y cirugía son las formas más promisorias en el tratamiento del cáncer. Sin embargo, hay conocimiento de otras técnicas de tratamiento, las llamadas prácticas alternativas, caracterizadas por la utilización de métodos no convencionales. Los efectos adversos de algunos medicamentos sintéticos y los síntomas de las enfermedades crónicas, como las crisis alérgicas, nos lleva a pensar que algunos métodos vengan a aumentar la calidad de vida de las personas y así nos dirige a las prácticas complementarias asociadas al tratamiento médico tradicional (3).

La OMS define planta medicinal como todo y cualquier vegetal que posee substancias que pueden ser utilizadas con finalidades terapéuticas o que sean precursores de fármacos semisintéticas (4). Con el paso de los años se han registrado varios procedimientos clínicos que utilizan las plantas medicinales tradicionales (5). El uso de plantas medicinales con fines terapéuticos es una práctica antigua, basada en el conocimiento del sentido común en un contexto histórico, representando así parte de la cultura de un pueblo y un conocimiento que es difundido de generación en generación, convirtiéndose de esta forma en una práctica muy utilizada en la salud humana. 
Es en la familia que se inicia la sociedad y en ella los individuos organizan conceptos y buscan la madurez a través de intercambios. La familia actúa no solo en el sentido de amparar física, emocional y socialmente a sus miembros, sino también esclareciendo lo que es mejor o peor para su crecimiento, cupiendo a ella la responsabilidad de proporcionar calidad de vida a los miembros (6). Y es entre las diferentes generaciones de la familia donde se da la transmisión del conocimiento relacionado con las terapias complementarias.

Este trabajo tiene por objetivo relatar el uso de plantas como terapia complementaria, por pacientes con cáncer que realizan radioterapia en un ambulatorio de propiedad de una Universidad Pública en el sur de Río Grande do Sul

\section{MATERIALES Y MÉTODOS}

Se trata de un recorte del proyecto de investigación "Intervenciones de Enfermería con Clientes Oncológicos y Familiares en un Ambulatorio de Radioterapia de la UFPel" coordinada por la Dra. Eda Schwartz , Apoyo FAPERGS /PROADE -3 número 05/2279.2.

Fue realizada una investigación de abordaje cualitativo, para la profundización y extensión de la comprensión de una situación. Su criterio, por lo tanto, no es numérico, mas se preocupa con un nivel de realidad que no puede ser cuantificada ${ }^{(7)}$. El carácter de la investigación es descriptivo, pretende describir con precisión los hechos y fenómenos de determinada realidad ${ }^{(8-9)}$, con clientes oncológicos y sus familiares los cuales frecuentaban un centro de radioterapia.

El proyecto fue aprobado por la Comisión de Ética de la Universidad en cuestión (n`028/06), según los principios y la resolución del Consejo Nacional de Salud 196/96. El instrumento utilizado fue una entrevista semiestructurada, la cual combina preguntas abiertas y cerradas, donde el entrevistado tiene la posibilidad de discurrir sobre el tema propuesto, sin respuestas o condiciones prefijadas por el investigador ${ }^{(6)}$, conteniendo, entre otras, cuestiones relacionadas a la enfermedad y las terapias complementarias utilizadas en el enfrentamiento de la misma. Las entrevistas fueron aplicadas en el período de Enero a Diciembre de 2006 y fueron entrevistados 20 clientes y sus respectivos familiares que utilizaban el servicio de radioterapia vinculado a una Universidad Pública de la ciudad de Pelotas-RS. Las entrevistas fueron grabadas y después transcriptas en la íntegra para posterior análisis. Los participantes fueron nombrados por medio de números arábigos, conforme la secuencia de las entrevistas.

El análisis de los datos ocurrió hasta Julio de 2007, los mismos fueron organizados en temas. Los datos cualitativos son importantes en la construcción del conocimiento y ellos pueden permitir el inicio de una teoría o su reformulación. El principio general es de que todos los datos deben ser articulados con la teoría ${ }^{(7)}$.

En este artículo serán discutidas solo las respuestas relativas a las prácticas terapéuticas complementarias utilizadas por pacientes oncológicos durante el recorrido de la enfermedad y el debido tratamiento.

\section{RESULTADOS Y DISCUSIÓN}

Durante las entrevistas aparecieron resultados que muestran la amplia utilización de terapias complementarias por pacientes portadores de cáncer que realizan radioterapia, entre las terapias citadas surgió la utilización de plantas medicinales para auxiliar en el tratamiento del cáncer. 
Algunas charlas remiten al empleo de la plantas:

Té yo tomo, los que hay en casa. (Cliente 01)

A veces uno llega allá no, un té conocido no, hay gente allá afuera que sale de casa en casa vendiendo, y ahí uno compra un tesito aquí, otro allí [...] (Cliente05)

Otros pacientes relataron los variados tipos de plantas utilizadas en el transcurso de la enfermedad, creyendo que esas son una forma de potencializar el tratamiento radioterápico:

Tratamiento natural, con hierbas. Yo tomo cavalinha, cocão, ipê roxo y también cáscara de nueces molidas, hago té, cáscara de nueces yo conservo helado en la heladera, en los intervalos, en verano tomo mucho [...] es indicado para eso ahí que cura cáncer [...] yo le dije al Doctor, yo estoy haciendo ese tratamiento, no, todo bien puede hacer, solo no vamos a perder el contacto, vamos a mantener relaciones, charlas y consultas [...](Cliente 07)

Pomada yo siempre usaba, benzedeira, babosa, yo sé que últimamente estaba usando la pomada novacort, mas yo usé varias, decían, usa esto, esto es bueno... mas yo lavé con té, babosa, té de marcela, camomila... yo creía en lo que me decían que era bueno y usaba[...] (Cliente10)

Se sabe que las plantas medicinales poseen nombres populares diversos en las diferentes regiones del Brasil. Inclusive dentro del mismo Estado pueden ser conocidas por nombres diferentes; para facilitar el reconocimiento de esas plantas, cito a lo largo del trabajo el nombre popular de la planta, atribuido por los pacientes, y también el nombre científico por la cual ella es conocida.

Los entrevistados relataron que utilizaron diferentes especies de plantas como terapias complementarias para tratamiento del cáncer. La literatura apunta que algunas de esas plantas realmente tienen efecto antitumoral comprobado, mientras que otras no lo tienen hasta la presente constatación científica.

En estudio realizado entre 2000 y 2001, que trata del uso de plantas medicinales por los pacientes con cáncer de hospitales de la red pública de salud en João Pessoa (PB), así como en el presente estudio, constataron la utilización de babosa (Aloe sp), camomila (Matricaria chamomilla) e ipê-roxo (Tabebuia avelanedae) por los portadores de cáncer. Los autores de esa investigación relatan la existencia de otras investigaciones que evaluaron las actividades antitumorales de esas plantas. Fueron consultadas 26 especies de Aloe y evaluadas las propiedades antineoplásicas, siendo todas activas cuanto a su acción farmacológica. Además de esa función se destaca acciones laxativas, hepatoprotetora, antiinflamatoria, cicatrizante, emoliente, hidratante y protectora de la piel contra rayos solares, antihemorroidal, antihelmíntica y antibacteriana. Sin embargo, conviene resaltar que el uso interno prolongado de la babosa lleva a la pérdida de minerales esenciales, pudiendo causar también dolores abdominales, diarrea sanguinolenta, hemorragias gástricas y nefritis. Con relación a camomila los autores citan tres estudios preclínicos en cuanto a la evaluación de la acción de la planta, en los que se mostró inactiva en cuanto a propiedades antitumorales. Ya el ipê-roxo fue citado como efectivo en la actividad antineoplásica, según ensayos clínicos ${ }^{(10)}$. 
Otros estudios comprueban que el uso de las cáscaras del ipê-roxo bajo la forma de té ofrece alguna actividad contra algunos tipos de cáncer, siendo tóxico en dosis elevadas, llevando a la pérdida de peso, anorexia y diarrea. El aislamiento de la sustancia lapachol, presente en esa planta, en ensayos farmacológicos, presentó actividad antineoplásica, mas pese a que algunos aislados de esa planta hayan mostrado propiedad citotóxica para tumores humanos, no se puede garantizar que el uso de sus preparaciones caseras tenga suceso en el tratamiento del cáncer. Los mismos autores citan estudios realizados in vitro muestran que extractos de flores de la planta de macela (Achyrocline satureioides) inhibieron en $67 \%$ el desarrollo de células cancerígenas ${ }^{(11)}$.

Existe una tendencia mundial de crecimiento de prácticas no convencionales en el campo de la salud, y de legislación para su integración en los sistemas nacionales de salud ${ }^{(12)}$. Sin embargo, es importante destacar que en Brasil existe deficiencia en investigaciones científicas que relaten experiencias con plantas medicinales y sus propiedades en salud humana, así se hacen necesarias iniciativas que tornen profesionales de la salud capacitados para fortalecer orientaciones al respecto del uso seguro de plantas medicinales a pacientes que frecuentan Unidades de Salud, principalmente aquellos portadores de enfermedades crónicas como cáncer que realizan tratamientos medicamentosos y utilizan terapias complementarias. Actualmente, muchas plantas están siendo estudiadas científicamente, adquiriendo poco a poco su espacio en el medio académico y en la actuación de los profesionales de salud, inclusive en el campo de la enfermería.

La publicación de la Portaría no. 971 configura el marco decisivo del proceso de institucionalización de estos abordajes en el Sistema Único de Salud ${ }^{(12)}$.

Se nota la necesidad de entrenamientos para los profesionales de la salud, así como de la enfermería, para tratar con tales prácticas. De esta manera, podrán fortalecer orientaciones adecuadas y esenciales a los pacientes sobre el uso de las terapias alternativas, a fin de evitar posibles riesgos y agravios a la salud por la utilización inadecuada de tales prácticas (3).

Se pueden observar experimentos con terapias complementarias, bien como plantas medicinales en el ámbito preventivo y curativo, pero aún no se ha logrado suficiencia tanto en el campo de la discusión como en la práctica concreta para garantizar su legitimidad, siendo muchas veces apenas referido su uso popular ${ }^{(13-14)}$. Eso puede ocurrir por la no incorporación de forma efectiva de ese conocimiento en el currículo formador, ahí se hace necesaria e importante la reflexión sobre su aplicabilidad, tanto en la formación académica, como en la práctica profesional de los enfermeros ${ }^{(13-15)}$.

\section{CONCLUSIÓN}

En el presente trabajo, se constató que los pacientes con cáncer no utilizan solamente el tratamiento convencional indicado por el médico, sino que también, en la mayoría de las veces, procuran otros tipos de terapias complementarias como, por ejemplo, las plantas medicinales. Se percibe que al buscar las terapias complementarias buscan algo que haga potencializar su tratamiento, adquiriendo autonomía en su proceso de recuperación y creyendo así en la cura. Son evidenciados algunos cuidados que deben ser tomados en la utilización de la terapia citada, que por ser natural, aparenta ser inofensiva. Es importante destacar que las plantas, algunas veces, pueden traer resultados negativos si se utilizan de forma equivocada. De ahí la importancia de informar a la población que hace uso de tales terapias al respecto de propiedades tóxicas de algunas plantas. 
En este estudio, constatamos que los profesionales de salud que trabajan directamente con portadores de cáncer deben estar cualificados para ofrecer informaciones seguras al respecto de las terapias complementarias. Reflexionar sobre esas implicaciones en el cuidado de enfermería se convirtió en algo urgente en la profesión; hay que democratizar y relativizar el empleo de esas terapias en el sentido de una acción compartida e interdisciplinaria en el cuidado a la salud, en el caso de la enfermería, como una extensión de su práctica de cuidar.

\section{REFERÊNCIAS}

1. Brasil. Ministério da Saúde. INCA. Estimativa de incidência de câncer no Brasil para 2008. Rio de Janeiro; 2008. Disponível: em http://www.inca.gov.br.

2. Casarin ST, Heck RM, Schwartz E. O uso de práticas terapêuticas alternativas, sob a ótica do paciente oncológico e sua família. Revista Família, Saúde e Desenvolvimento. 2005; 7: 24-31.

3. Jacondino CB, Amestoy SC, Thofehrn MB. A utilização de terapias alternativas por pacientes em tratamento quimioterápico. Revista Cogitare. 2008 Jan-Mar: 61-66.

4. Bulletin of the World Health Organization. Regulatory situation of herbal medicines. A worldwide review, Geneva, 1998.

5. Veiga JVF, Pinto AC, Maciel MAM. Plantas Medicinais: Cura Segura? Revista Química Nova. 2005 (28): 519-528.

6. Negrelli MED, Marcon SS. Família e criança surda. Ciência, Cuidado e Saúde. 2006 Jan/Jun; v.5, n. 1, 98-107.

7. Mynaio MCS. O desafio do conhecimento: pesquisa qualitativa em saúde. $2^{a}$ edição. São Paulo: Hucitec, 1993. 269 p.

8. Cervo AL, Bervian PA. Metodologia Científica. São Paulo: MC Graw-Hill, 1983.

9. Triviños, ANS. Introdução à pesquisa em ciências sociais: a pesquisa qualitativa em educação. São Paulo: Atlas, 1992.

10. Araújo EC, Oliveira RAG, Coriolano AT, Araújo EC. Uso de plantas medicinais pelos pacientes com câncer de hospitais da rede pública de saúde em João Pessoa (PB). Revista Espaço para Saúde. 2007 Jun; 8 (2):44-52.

11. Lorenzi H, Matos FJA. Plantas medicinais no Brasil: nativas e exóticas. São Paulo: Nova Odessa, 2002. 512 p.

12. Brasil. Ministério da Saúde. Práticas Integrativas e Complementares em Saúde: uma realidade no SUS. Revista Brasileira de Saúde da Família. Ano X, edição especial. BrasíliaDF; 2008.

13. Alvim NAT, Ferreira MA, Cabral IE, Almeida Filho AJ. O uso de plantas medicinais como recurso terapêutico: das influências da formação profissional às implicações éticas e legais de sua aplicabilidade como extensão da prática de cuidar realizada pela enfermeira. Revista Latino Americana de Enfermagem. 2006 Mai-Jun; 14(3).

14. Domínguez MCR, Pérez VR, Trujillo JMG. Procedimiento de enfermería: "curas de heridas agudas y crónicas con el filete de aloe vera". Revista Enfermería Global. Mayo 2007; 10: 1-13.

15. Blanca Gutiérrez JJ, Alvarez Nieto C, Alba Fernández CM. Terapias complementarias en la escuela de enfermería. Revista Enfermería Global. Mayo 2007; 10: 1-8.

ISSN 1695-6141

@ COPYRIGHT Servicio de Publicaciones - Universidad de Murcia 